\title{
TRANSVERSALS AS GENERATING SETS IN FINITELY GENERATED GROUPS
}

\author{
JACK BUTTON, MAURICE CHIODO, MARIANO ZERON-MEDINA LARIS
}

\begin{abstract}
We explore transversals of finite index subgroups of finitely generated groups. We show that when $H$ is a subgroup of a rank $n$ group $G$ and $H$ has index at least $n$ in $G$ then we can construct a left transversal for $H$ which contains a generating set of size $n$ for $G$; this construction is algorithmic when $G$ is finitely presented. We also show that, in the case where $G$ has rank $n \leq 3$, there is a simultaneous left-right transversal for $H$ which contains a generating set of size $n$ for $G$. We finish by showing that if $H$ is a subgroup of a rank $n$ group $G$ with index less than $3 \cdot 2^{n-1}$, and $H$ contains no primitive elements of $G$, then $H$ is normal in $G$ and $G / H \cong C_{2}^{n}$.
\end{abstract}

\section{INTRODUCTION}

Let $H$ be a subgroup of $G$ (written $H<G$ ). A left (resp. right) transversal for $H$ in $G$ is a choice of exactly one representative from each left (resp. right) coset of $H$. A left-right transversal for $H$ is a set $S$ which is simultaneously a left, and a right, transversal for $H$ in $G$. The existence of a left or right transversal for subgroups of finitely generated groups is clear, and for general groups this condition is equivalent to Axiom of Choice ([1, Theorem 2.1]). However, it is not obvious that a left-right transversal always exists. We gave a short proof of this in [3] for the case where $H$ is of finite index, as well as a brief historical discussion of this result.

Transversals are natural objects of study, especially when the index $[G: H]$ is finite. Moreover, finding generating sets for a group $G$ is a well known problem in the case when $G$ is finitely generated. Therefore we ask: given a finitely generated group $G$ and a finite index subgroup $H$ of $G$, is there a (say) left transversal for $H$ in $G$ which also generates $G$ ? Jain asked Cameron this question under the added assumption that $G$ is a finite group and $H$ is corefree in $G$, meaning that $\operatorname{core}_{G}(H)=\{e\}$ (where core $G(H):=\bigcap_{g \in G} g^{-1} H g$ ). Cameron showed in [4] that in this case a generating left transversal always exists (see also [5, Problem 100]). The proof is short but relies on a result [13] of Whiston on minimal generating sets (ones where no proper subset generates) of the symmetric group which uses the classification of finite simple groups (CFSG).

2010 AMS Classification: 20E99, 20F05

Keywords: Transversals, generating sets, finite index subgroups, primitive elements.

The second author was partially funded by the Italian FIRB "Futuro in Ricerca" project RBFR10DGUA_002 and the Swiss National Science Foundation grant FN PP00P2-144681/1.

The third author did part of this work while writing his thesis at the University of Cambridge, supported by the Mexican National Council for Science and Technology.

Date: May 21, 2015. 
A necessary condition for a subgroup $H<G$ to possess a generating left transversal is that $[G: H]$ must be at least $d(G)$ (the rank of $G$ ), defined to be the minimal number of generators for the finitely generated group $G$. In Theorem 3.7 we show that this condition is also sufficient, for $G$ any finitely generated group and $H$ any subgroup of finite index. We then try to strengthen this result by examining whether $[G: H] \geq d(G)$ implies that there exists a leftright transversal for $H$ that generates $G$. We have not managed to establish this in general but we have shown in Theorem 3.11 that it is true if $d(G) \leq 3$.

Our main method of proof in Section 3 is a new technique which we call shifting boxes. It involves using the transitive action of a group $G$ on the set of left (or right) cosets of a subgroup $H<G$ to systematically apply Nielsen transformations to a generating set of $G$, such that the resulting generators lie inside (or outside) particular desired cosets of $H$. We have found this technique to be very intuitive for developing proofs. Many of our results can be reduced to the case of subgroups of free groups (Proposition 3.12). An element of a rank $n$ group $G$ is primitive if it lies in some generating set of size $n$ for $G$. The location of primitive elements relative to cosets of subgroups is already an area of interest. Parzanchevski and Puder [11] show that if $w \in F_{n}$ is a non-primitive element then there is a finite index subgroup $H<F_{n}$ such that the coset $w H$ does not contain any primitive elements. Taking $w=e$ gives a finite index subgroup containing no primitive elements.

By applying the technique of shifting boxes developed in Section 3, we show in Theorem 4.4 that, if $G$ is a rank $n$ group, then the only subgroup of $G$ with index less than $3 \cdot 2^{n-1}$ that can contain no primitive elements is $[G, G] G^{2}$, and even then this only occurs when $G /\left([G, G] G^{2}\right) \cong C_{2}^{n}$. This gives an exponential lower bound on the index of subgroups which contain no primitive elements. We first announced many of the results of this paper in [2].

Acknowledgements: We wish to thank Rishi Vyas, Andrew Glass, Zachiri McKenzie and the anonymous referee for their useful comments on this work.

\section{Coset intersection GRAPHS}

A useful tool for studying the way left and right cosets interact, and obtaining transversals, is the coset intersection graph. In this section we re-state important results from our earlier work [3] on this concept. We denote the complete bipartite graph on $(m, n)$ vertices by $\mathbf{K}_{m, n}$.

Definition 2.1. Let $H, K<G$. We define the coset intersection graph $\Gamma_{H, K}^{G}$ as the graph with vertex set consisting of all left cosets of $H\left(\left\{l_{i} H\right\}_{i \in I}\right)$ and all right cosets of $K\left(\left\{K r_{j}\right\}_{j \in J}\right)$, where $I, J$ are index sets. If a left coset of $H$ and right coset of $K$ correspond, they are still included twice. Edges (undirected) are included whenever any two of these cosets intersect, and an edge between $a H$ and $K b$ (written $a H-K b$ ) corresponds to the non-empty set $a H \cap K b$.

Theorem 2.2. Let $H, K<G$. Then the graph $\Gamma_{H, K}^{G}$ is a disjoint union of complete bipartite graphs. Moreover, suppose that $[G: H]=n,[G: K]=m$. Then each connected component of $\Gamma_{H, K}^{G}$ is of the form $\mathbf{K}_{s_{i}, t_{i}}$ with $s_{i} / t_{i}=n / m$. 
Corollary 2.3. Let $H, K<G$. Suppose that $[G: H]=n$ and $[G: K]=m$, where $m \geq n$. Then there exists a set $T \subseteq G$ which is a left transversal for $H$ in $G$, and which can be extended to a right transversal for $K$ in $G$. If $H=K$ in $G$, then $T$ becomes a left-right transversal for $H$.

Under the hypothesis of Theorem 2.2, we see that sets of $s_{i}$ left cosets of $H$ completely intersect sets of $t_{i}$ right cosets of $K$, with $s_{i} / t_{i}$ constant over $i$. With this in mind, another way of visualising $\Gamma_{H, K}^{G}$ is by the following simultaneous double-partitioning $G$ : draw left cosets of $H$ as columns, and right cosets of $K$ as rows, partitioning $G$ into irregular 'chessboards' denoted $C_{i}$, each with edge ratio $n: m$. Each chessboard $C_{i}$ corresponds to the connected component $\mathbf{K}_{s_{i}, t_{i}}$ of $\Gamma_{H, K}^{G}$, and individual tiles in $C_{i}$ correspond to the non-empty intersection of a left coset of $H$ and a right coset of $K$ (i.e., edges in $\mathbf{K}_{s_{i}, t_{i}}$ ). Corollary 2.3 would then follow by choosing one element from each tile on the leading diagonals of the $C_{i}$ 's. An example of chessboards is given in [3].

The chessboard pictorial representation of partitioning $G$ into left and right cosets is extremely useful in the analysis of transversals as generating sets carried out in the next section. Note that the union of all the elements of $G$ in a single chessboard gives a unique double coset $K g H$ in $G$, and that a single chessboard is simply a double-partitioning of a double coset $K g H$ into its respective left cosets of $H$ and right cosets of $K$.

\section{TRAnSVERsals AS GENERATING SETS}

We have developed a technique which we call shifting boxes which, for the sake of brevity, we will describe here as a systematic way to apply Nielsen transformations to a generating set of a group $G$, such that the resulting generators lie inside (or outside) particular desired cosets of a subgroup $H<G$. We can't 'shift' generators in/out of any coset we like, but we do have a substantial degree of control. For ease of notation, we will often refer to the coset $e H$ as the identity coset. We begin with the following definitions.

Definition 3.1. Let $G$ be a group, and $S:=\left(g_{1}, \ldots, g_{n}\right)$ a generating $n$-tuple of $G$ (where $n \in \mathbb{N}$ ), that is, an element of the direct product $G^{n}$ such that $\left\{g_{1}, \ldots, g_{n}\right\}$ generates $G$. A standard Nielsen move on $S$ is the replacement of some entry $g_{i}$ of $S$ with one of $g_{j} g_{i}, g_{j}^{-1} g_{i}, g_{i} g_{j}$ or $g_{i} g_{j}^{-1}$, where we must have $i \neq j$. A Nielsen move is defined to be either a standard Nielsen move or an extended Nielsen move, where the latter consists of either replacing an entry $g_{i}$ by its inverse, or transposing two entries $g_{i}$ and $g_{j}$ for $i \neq j$. Note that on applying any Nielsen move to $S$, the resulting $n$-tuple still generates $G$. Two generating $n$-tuples $S_{1}, S_{2}$ of $G$ are said to be Nielsen equivalent if they differ by a finite number of Nielsen moves.

Definition 3.2. Let $H<G$ be groups, and $S$ a generating $n$-tuple of $G$. We say a left coset $g H$ is full (with respect to $S$ ) if some entry of $S$ lies in $g H$, otherwise we say $g H$ is empty (with respect to $S$ ). To save on notation, we usually suppress the term 'with respect to $S$ ' when there is no ambiguity. 
We now give several techniques, which we rely on heavily for our main results. Note that in this section we prove our results under very general conditions, and all techniques are (for now) existential.

Definition 3.3. Let $H<G$ be groups. A $n$-tuple $S^{\prime}$ with entries in $G$ is said to be left-cleaned if all of its entries lie in distinct left cosets of $H$, apart from $e H$ which may contain many entries of $S^{\prime}$.

Lemma 3.4. Let $H<G$ be groups, and $S$ a generating $n$-tuple of $G$. Then there is a left-cleaned generating $n$-tuple $S^{\prime}$ of $G$, Nielsen equivalent to $S$.

Proof. We call the following process left-cleaning an $n$-tuple. Let $S=\left(g_{1}, \ldots, g_{n}\right)$. We can assume that there is $g_{i}, g_{j}$ with $i \neq j$ both lying in the same non-identity left coset of $H$, so that $g_{i} H=g_{j} H \neq e H$. Then $g_{j}^{-1} g_{i} \in H$ so we can apply the standard Nielsen move on $S$ which replaces $g_{i}$ with $g_{j}^{-1} g_{i}$ to obtain $S_{1}$. Then $S_{1}$ has fewer entries lying in this left coset of $H$, and the same number in all other non-identity left cosets. Iterating this procedure and then moving to other non-identity left cosets, we eventually reach a left-cleaned $n$-tuple $S^{\prime}$.

Lemma 3.5. Let $H<G$ be groups, and $S$ a generating $n$-tuple of $G$. If there exists at least one empty left coset of $H$, then there are entries $s_{j}, s_{k}$ of $S$ (possibly the same entry) and $\epsilon \in\{ \pm 1\}$ such that $s_{j}^{\epsilon} s_{k} H$ is an empty left coset. That is, there is some full left coset of $H$ which is taken to some empty left coset of $H$ by left multiplication under some entry of $S$ or its inverse.

Proof. Recall that $G$ acts transitively on the set of left cosets by left multiplication. Assume that no entry of $S$ or its inverse sends a full left coset to an empty left coset. Then, as the entries of $S$ generate $G$, the collection of full left cosets is invariant under this action. Seeing as there exists at least one empty left coset, this contradicts the transitive action of $G$.

Lemma 3.6. Let $H<G$ be groups, and $S$ a generating $n$-tuple of $G$. Suppose that at least one entry of $S$ lies in $H$, and moreover that there exists an empty left coset of $H$ with respect to $S$. Then there is a finite sequence of Nielsen moves on some entry $s$ of $S$ which is contained in $H$ such that $s$ is taken into an empty left coset of $H$.

Proof. By Lemma 3.5 there are (possibly identical) entries $s_{1}, s_{2}$ of $S$, and $\epsilon \in\{ \pm 1\}$, with $s_{j}^{\epsilon} s_{k} H$ an empty left coset of $H$ with respect to $S$. We consider all possible cases:

1. The case $s_{j}, s_{k} \in H$ never occurs, as then $s_{j}^{\epsilon} s_{k} H=H$ which is a full left coset by hypothesis.

2. In the case $s_{j} \notin H, s_{k} \in H$, the subcase $s_{j}^{+1} s_{k} H$ can't occur, as then $s_{j}^{+1} s_{k} H=$ $s_{j}^{+1} H$ which is clearly full. In the subcase $s_{j}^{-1} s_{k} H$, we replace $s_{k}$ with $s_{j}^{-1} s_{k}$ lying in $s_{j}^{-1} s_{k} H$ which is empty.

3. In the case $s_{j} \in H, s_{k} \notin H$, we replace $s_{j}$ with $s_{j}^{\epsilon} s_{k}$, as $s_{j}^{\epsilon} s_{k} H$ is empty.

4. In the case $s_{j}, s_{k} \notin H$, take some $s_{i} \in H$ and replace $s_{i}$ with $s_{j}^{\epsilon} s_{k} s_{i}$, which lies in $s_{j}^{\epsilon} s_{k} s_{i} H=s_{j}^{\epsilon} s_{k} H$ which is empty. As $s_{i}$ is a different entry from $s_{j}$ and $s_{k}$, this is a composition of two Nielsen moves on the entry $s_{i}$ (even if $s_{j}=s_{k}$ ).

We call this replacement process a left-extraction of an entry of $S$ from $H$. 
Using these techniques, we state the condition below for a finite index subgroup of a group to possess a left transversal which generates the whole group. For simplicity, when $S$ is an $n$-tuple, we write $\tilde{S}$ for the set of entries of $S$.

Theorem 3.7. Let $G$ be a finitely generated group, and $H$ a subgroup of finite index in $G$. Then the following are equivalent:

1. $[G: H] \geq d(G)$.

2. There exists a left transversal $T$ for $H$ in $G$ which contains a generating set $X$ for $G$ of size $|X|=d(G)$.

Proof. That $2 \Rightarrow 1$ is immediate. We show $1 \Rightarrow 2$ :

Let $n=d(G)$, and let $S$ be a generating $n$-tuple for $G$. Use Lemma 3.4 to produce an $n$-tuple $S^{\prime \prime}$ Nielsen-equivalent to $S$ which is left-cleaned. Now repeatedly apply Lemma 3.6 to begin left-extracting elements from inside $H$ (thus Nielsen-transforming $\left.S^{\prime \prime}\right)$. As $n \leq[G: H]$, we can keep left-extracting until we reach $S^{\prime}$ which is Nielsen-equivalent to our original $S$, and for which no two entries of $S^{\prime}$ lie in the same left coset of $H$. Now simply choose one element from each left coset of $H$ which is empty with respect to $S^{\prime}$, and add these to $\tilde{S}^{\prime}$ to form the set $T$. Then $T$ is a left transversal for $H$, and contains $\tilde{S}^{\prime}$.

A slight variant of the above proof also shows the following result: when $[G: H] \leq d(G)$ then there is a generating set for $G$ of size $d(G)$ which contains a set of left coset representatives for $H$.

Note that all preceeding definitions and results in this section carry over to right transversals, which for the sake of brevity we do not re-state explicitly.

For ease of writing, we will often refer to the overall process of cleaning and/or extracting elements (either left, or right) as shifting boxes, and will usually just write this follows by shifting boxes to mean that it follows by the process of cleaning and/or extracting elements. Our remarks in this section give sufficient conditions for cleaning and/or extracting to be algorithmic.

The most natural question to ask now is 'When does a finite index subgroup have a left-right transversal which generates the whole group?' This requires a deeper understanding of how cosets intersect, as discussed in Section 2. We urge the reader to consider the discussion of 'chessboards' given after Corollary 2.3 , and to consult [3] for an example. These are vital in proving what follows.

Let $H<G$ be groups, and $S$ a generating $n$-tuple of $G$. Then by Lemma 3.4 we can first perform a left-cleaning of $S$ to form $S^{\prime}$, followed by a right-cleaning of $S^{\prime}$ (which will remain left-cleaned) to obtain $S^{\prime \prime}$. It follows that $S$ and $S^{\prime \prime}$ are Nielsen-equivalent, and that $S^{\prime \prime}$ is both left-cleaned and right-cleaned.

Lemma 3.8. Let $H<G$ be of finite index in $G$, and $S$ a generating n-tuple of $G$. Then $S$ is left-right-cleaned if and only if one can draw chessboards for $H$ in $G$ with distinct entries of $S$ lying in distinct diagonal tiles of chessboards, except for the chessboard corresponding to the double coset $\mathrm{HeH}=\mathrm{H}$ which may contain several elements of $S$.

Proof. This is immediate from the fact that columns in chessboards correspond to left cosets of $H$, and rows correspond to right cosets. Thus, a column (resp. row) in the chessboards contains multiple entries of $S$ if and only if the corresponding left (resp. right) coset of $H$ contains multiple entries of $S$. 
Note that we can obtain a left-right transversal for $H$ by taking one element from each diagonal tile of each chessboard (Corollary 2.3). In fact, by left-rightcleaning and choosing an element from each unused diagonal we have:

Lemma 3.9. Let $H<G$ be of finite index in $G$, and $S$ a generating $n$-tuple of $G$ with at most one entry in $H$. If $S$ is left-right-cleaned, then there is a set $T$ containing all the entries of $S$ which is a left-right transversal for $H$ in $G$.

Proof. Given that columns in chessboards correspond to left cosets of $H$, and rows correspond to right cosets, we have that no column or row in any chessboard contains more than one entry from $S$. Thus we can re-arrange the positioning of the columns and rows in each chessboard so that the entries of $S$ are all in tiles which lie on leading diagonals. Now simply choose one element from each lead-diagonal tile which does not contain an entry of $S$, and add these to the set $S$ to form the set $T$. Then $T$ contains precisely one element from each lead-diagonal tile of each chessboard, and no other elements. Thus $T$ contains precisely one element in each left coset of $H$, and precisely on element in each right coset of $H$. So $T$ is our desired left-right transversal which contains $\tilde{S}$.

Combining our shifting boxes technique with the properties of the coset intersection graph from Theorem 2.2, we are able to show the following:

Theorem 3.10. Let $H<G$ be of finite index in $G$, and $S$ a generating $n$ tuple of $G$ with $n \leq[G: H]$. If $n \leq 3$ then there is a generating n-tuple $S^{\prime}$ Nielsen-equivalent to $S$, and a left-right transversal $T$ for $H$ in $G$ with $\tilde{S}^{\prime} \subseteq T$.

Proof. The case when $n=1$ is trivial. The case when $n=2$ is done as follows: Left-right-clean $S$ to form $S^{\prime}=(a, b)$. Clearly we can't have $a, b \in H$, or else $[G: H]<2$. So at most one of $a, b$ lies inside $H$. But then by Lemma 3.9 we can extend $\tilde{S}^{\prime}$ to a (generating) set $T$ which is a left-right transversal for $H$.

The case $n=3$ is complicated, and we consider several sub-cases. So, leftright-clean $S$ to form $S^{\prime}=(a, b, c)$. Clearly we can't have $a, b, c \in H$, or else $[G: H]<3$. If at most one of $a, b, c$ lies inside $H$ then we can apply Lemma 3.9 as before. So we are left with the case where two of $a, b, c$ lie inside $H$ (re-label them as $h_{1}, h_{2} \in H$ and $\left.g \notin H\right)$.

Case 1. $g^{2} \notin H g H \cup H$ (i.e., $g^{2}$ lies in a different chessboard to $g$ and $h_{1}, h_{2}$ ). Make the Nielsen moves $h_{1} \mapsto g^{2} h_{1}$; this clearly lies in the same left coset (and hence same chessboard) as $g^{2}$ (see Figure 1).
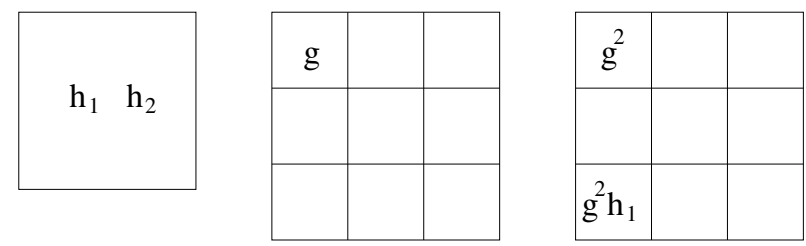

Figure 1

So now each of $g, h_{2}, g^{2} h_{1}$ lie in different chessboards, thus the triple $S^{\prime \prime}$ := $\left(g, h_{2}, g^{2} h_{1}\right)$ is left-right cleaned. As only $h_{2}$ lies inside $H$, we can use Lemma 
3.9 to extend $\tilde{S}^{\prime \prime}$ to a set $T$ which is a left-right transversal for $H$. Seeing as $T$ contains $\tilde{S}^{\prime \prime}$, then it generates $G$.

Case 2. $g^{2} \in H g H$ (i.e., $g^{2}$ lies in the same chessboard as $g$ ).

Clearly $g^{2} H \neq g H$ and $H g^{2} \neq H g$; otherwise we would have $g \in H$ which contradicts our initial hypothesis. So $g^{2}$ lies in a different left coset and different right coset to $g$ (i.e., in a different column and row to $g$ in $\mathrm{HgH}$ ). Consider $h_{1} g^{2}$ and $h_{2} g^{2}$ (which both lie in the same right coset as $g^{2}$, and hence in a different right coset to $g$ ). If $h_{i} g^{2} H \neq g H$ for some $i \in\{1,2\}$, then make the Nielsen moves $h_{i} \mapsto h_{i} g^{2}$ which lies in a different left and different right coset to $g$ (but in the same chessboard) (see Figure 2).
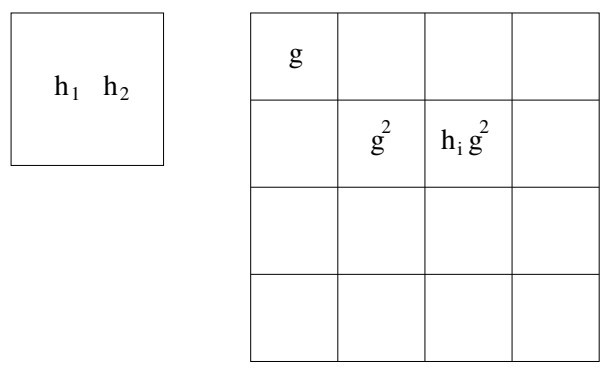

FIGURE 2

If on the other hand $h_{1} g^{2} H=h_{2} g^{2} H=g H$, then $h_{2}^{-1} h_{1} g^{2} H=g^{2} H$ and so we make the Nielsen moves $h_{1} \mapsto h_{2}^{-1} h_{1} g^{2}$ which lies in a different left and different right coset to $g$ (but in the same chessboard) (see Figure 3).
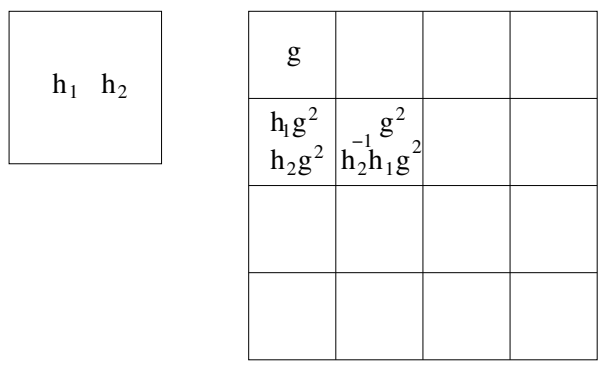

FIGURE 3

Either way, we now have a triple $S^{\prime \prime}$ for which, after permutation of some rows and columns, has entries which lie along diagonal tiles of the chessboards. We conclude as in case 1 .

Case 3. $g^{2} \in H$.

By the transitivity of the action of $G$ on left (and right) cosets of $H$, there must be some $h_{i}(i \in\{1,2\})$ and some $\epsilon \in\{ \pm 1\}$ with $h_{i}^{\epsilon} g H \neq g H$, and similarly some $h_{j}(j \in\{1,2\})$ and some $\delta \in\{ \pm 1\}$ with $H g h_{j}^{\delta} \neq H g$. If $i \neq j$, then we make the Nielsen move $h_{i} \mapsto h_{i}^{\epsilon} g$ followed by the Nielsen move $g \mapsto g h_{j}^{\delta}$ (see Figure 4).

If on the other hand $i=j$ (say $i=j=1$, without loss of generality), then consider the element $h_{2} g h_{1}^{\delta}$. If $h_{2} g h_{1}^{\delta} H \neq g H$, then $h_{2} g h_{1}^{\delta}$ lies in a different 


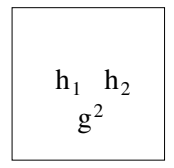

\begin{tabular}{|c|c|c|c|}
\hline $\mathrm{g}$ & & $\mathrm{h}_{\mathrm{i}}^{\mathrm{g}} \mathrm{g}$ & \\
\hline & & & \\
\hline $\mathrm{gh}_{\mathrm{j}}{ }^{\delta}$ & & & \\
\hline & & & \\
\hline
\end{tabular}

FIGURE 4

left coset and different right coset to $g$, and so we make the Nielsen moves $h_{2} \mapsto h_{2} g h_{1}^{\delta}$ (see Figure 5).

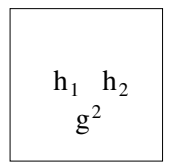

\begin{tabular}{|c|l|l|l|}
\hline $\mathrm{g}$ & & $\mathrm{h}_{1}^{\varepsilon} \mathrm{g}$ & \\
\hline & & & \\
\hline $\mathrm{gh}_{1}^{\delta}$ & $\mathrm{h}_{2} \mathrm{gh}_{1}^{\delta}$ & & \\
\hline & & & \\
\hline
\end{tabular}

FIGURE 5

If however $h_{2} g h_{1}^{\delta} H=g H$, then $h_{2} g h_{1}^{\delta}$ lies in a different left coset and different right coset to $h_{1}^{\epsilon} g$, and so we make the Nielsen moves $h_{2} \mapsto h_{2} g h_{1}^{\delta}$ followed by $g \mapsto h_{1}^{\epsilon} g$ (see Figure 6).

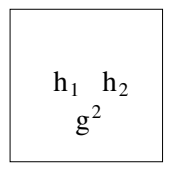

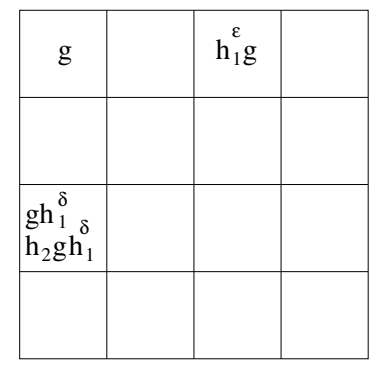

\section{FiguRE 6}

In all the subcases considered here, we end up with a triple $S^{\prime \prime}$ for which, after permutation of some rows and columns, has entries which lie along diagonal tiles of the chessboards. We conclude as in case 1.

We note that all previous results in this section are algorithmic, in that we have explicitly constructed the relevant transversals and generating sets.

Theorem 3.11. Let $G$ be a group with $d(G) \leq 3$, and $H$ a subgroup of finite index in $G$. Then the following are equivalent: 
1. There exists a left-right transversal $T$ for $H$ in $G$ with $\langle T\rangle=G$.

2. $[G: H] \geq d(G)$.

Proof. That $1 \Rightarrow 2$ is immediate; that $2 \Rightarrow 1$ can be seen from Theorem 3.10.

This leads us to pose the following question:

Question 1. Does Theorem 3.11 hold if we change the hypothesis ' $d(G) \leq 3$ ' to ' $d(G)$ finite'?

We have not yet been able to extend the proof of Theorem 3.10 to the case $n \geq 4$, as the number of scenarios to consider becomes very large and complex. However, we believe this should be possible, with some sort of more general technique of left-right-extraction.

We note that, in any extension of Theorem 3.11 to groups needing more than three generators, we need only consider free groups, as the following shows:

Proposition 3.12. Theorem 3.11 holds for all finite rank groups (and not just groups of rank at most 3) if and only if it holds for all finite rank free groups.

Proof. Suppose Theorem 3.11 holds for all $F_{n}$. Let $G$ be a group with $d(G)=n$, and $H<G$ have index $[G: H]=k \geq n$. Then there is a surjection $f: F_{n} \rightarrow G$, and it is a standard fact that the preimage $f^{-1}(H)$ also has index $k$ in $F_{n}$. By hypothesis, there is a left-right transversal $T$ of $f^{-1}(H)$ which generates $F_{n}$; it follows that $f(T)$ is a left-right transversal of $H$ which generates $G$.

\section{An APPLICATION OF SHIFTING BOXES: FINDING PRIMITIVE ELEMENTS}

Recall that a primitive element of a finite rank free group $F_{n}$ is one which lies in some generating set of size precisely $n$, which is equivalent to being an element of a free basis for $F_{n}$. If $G$ is an arbitrary group of finite rank $n$, then we say a primitive element in $G$ is an element lying in some generating set of size $n$ for $G$. This coincides with the definition of primitive elements in $F_{n}$.

An obvious question to ask is which subgroups of $F_{n}$ (or more generally, rank $n$ groups) contain a primitive element (we can ask this for both finite and infinite index subgroups). We first consider the case of normal subgroups.

The following is immediate by considering the image under the quotient map of a generating set of minimal size containing the relevant primitive element:

Lemma 4.1. Let $G$ be a group of finite rank $n$, and $N$ a normal subgroup of $G$. If $N$ contains some primitive element of $G$, then $d(G / N)<n$.

The converse statement is not true, even in the special case that $G=F_{n}$, as was shown in $[7,9]$. It is currently open if $N$ has finite index and here we briefly mention the connection with product replacement graphs. More can be found in the survey [10] of Pak which contains a range of references.

Given a finitely generated group $G$ and an integer $n \geq d(G)$, the product replacement graph $\Gamma_{n}(G)$ has vertices the generating $n$-tuples of $G$ with edges between two vertices if one is the image of another under a standard Nielsen move. A big area of study in this topic is the connectivity of $\Gamma_{n}(G)$. It can happen that $\Gamma_{n}(G)$ is disconnected when $n=d(G)$ (for instance finite abelian groups) but no example is known of a finite group $G$ and an integer $n>d(G)$ 
where $\Gamma_{n}(G)$ is disconnected. The relation with primitive elements is that if $N$ is a normal subgroup of $F_{n}$ containing no primitive element but $G=$ $F_{n} / N$ has $d(G)<n$ then $\Gamma_{n}(G)$ is disconnected. Hence there are examples of infinite finitely generated groups $G$ with $\Gamma_{n}(G)$ disconnected by $[7,9]$, but a normal subgroup $N$ of finite index containing no primitive element and with $d\left(F_{n} / N\right)<n$ would give rise to a finite group $G$ and integer $n>d(G)$ with $\Gamma_{n}(G)$ disconnected; the existence of which is currently unknown.

Our shifting boxes technique enables us to explore the location of primitive elements relative to cosets of finite index subgroups, in the following ways. We write $[n]$ for the set of integers $\{1, \ldots, n\}$, and $X \Delta Y$ for symmetric difference.

Lemma 4.2. Let $G$ be any group with $d(G)=n$, and $H$ a subgroup of finite index in $G$ with $[G: H]<3 \cdot 2^{n-1}$. If $H$ contains no primitive elements of $G$, then $H$ contains the square of every primitive element of $G$.

Proof. Let $S=\left(g_{1}, \ldots, g_{n}\right)$ be any left-cleaned generating $n$-tuple for $G$. For any $\varnothing \neq M=\left\{i_{1}, \ldots, i_{k}\right\} \subseteq[n]$, ordered so $i_{1}<\ldots<i_{k}$, define the unique word $w_{M}:=g_{i_{k}} g_{i_{k-1}} \cdots g_{i_{1}}$. Define the disjoint sets of words $A:=\left\{w_{M} \mid \varnothing \neq M \subseteq\right.$ $\{2, \ldots, n\}$ or $M=\{1\}\}, B:=\left\{w_{M} g_{1} \mid \varnothing \neq M \subseteq[n]\right\}$. Now set $T:=A \sqcup B$, and thus $|T|=3 \cdot 2^{n-1}-1$. By construction, the only element in $T$ which might not be primitive is $g_{1}^{2}$, and the rest are primitive by Nielsen transformations: for any $w_{M} g_{1} \in B$ with $M \neq\{1\}$ we take some $1 \neq i \in M$ and perform the Nielsen transformation $g_{i} \mapsto w_{M} g_{1}\left(g_{i}\right.$ appears precisely once in $\left.w_{M} g_{1}\right)$, a similar argument works for any $w_{M} \in A$.

We claim that, for any pair of distinct words $x, y \in T$, if $x H=y H$ then either $H$ contains a primitive element or $g_{1}^{2} \in H$ (possibly both). We consider all cases: 1. $x, y \in A$, so $x=w_{M}, y=w_{M^{\prime}}$, with $M \neq M^{\prime}$. Then $w_{M}^{-1} w_{M^{\prime}} \in H$ is primitive as there is some $i \in M \Delta M^{\prime}$, so Nielsen transform $g_{i} \mapsto w_{M}^{-1} w_{M^{\prime}}$.

2. Precisely one of $x, y$ lie in $A$ (say $x \in A$ ), so $x=w_{M}$ and $y=w_{M^{\prime}} g_{1}$. We consider all subcases: 2A) $1 \notin M, M^{\prime}$. In this case, $w_{M}^{-1} w_{M^{\prime}} g_{1} \in H$ is primitive (Nielsen transform $g_{1} \mapsto w_{M}^{-1} w_{M^{\prime}} g_{1}$ ). 2B) $1 \in M^{\prime}, 1 \notin M$, and $M^{\prime}=M \cup\{1\}$. In this case, $w_{M}^{-1} w_{M^{\prime}} g_{1}=g_{1}^{2} \in H$. 2C) $1 \in M^{\prime}, 1 \notin M$, and there is some $1<j \in M \Delta M^{\prime}$. In this case, $w_{M}^{-1} w_{M^{\prime}} g_{1} \in H$ is primitive (Nielsen transform $\left.g_{j} \mapsto w_{M}^{-1} w_{M^{\prime}} g_{1}\right)$. 2D) $M=\{1\}$. If $M^{\prime}=\{1\}$ then $w_{M}^{-1} w_{M^{\prime}} g_{1}=g_{1} \in H$ is primitive. Otherwise, there is some $1 \neq j \in M^{\prime}$, in which case $w_{M}^{-1} w_{M^{\prime}} g_{1} \in H$ is primitive (Nielsen transform $g_{j} \mapsto w_{M}^{-1} w_{M^{\prime}} g_{1}$ ).

3. $x, y \in B$. In this case, $x=w_{M} g_{1}, y=w_{M^{\prime}} g_{1}\left(M \neq M^{\prime}\right)$. If there is some $1 \neq i \in M \Delta M^{\prime}$, then the element $g_{1}^{-1} w_{M}^{-1} w_{M^{\prime}} g_{1} \in H$ is primitive. Otherwise, $M^{\prime}=M \cup\{1\}$ (or $M=M^{\prime} \cup\{1\}$ ), in which case $g_{1}^{-1} w_{M}^{-1} w_{M^{\prime}} g_{1}=g_{1}^{-1} w_{M}^{-1} w_{M} g_{1} g_{1}=$ $g_{1} \in H$ (or $g_{1}^{-1} w_{M}^{-1} w_{M^{\prime}} g_{1}=g_{1}^{-1} g_{1}^{-1} w_{M^{\prime}}^{-1} w_{M^{\prime}} g_{1}=g_{1}^{-1} \in H$ ) is primitive.

Suppose that $H$ contains no primitive element. Since $|T|=3 \cdot 2^{n-1}-1 \geq[G: H]$ then either two elements of $T$ lie in the same coset (so by the claim above, $g_{1}^{2} \in H$ as $H$ contains no primitive element), or one element of $T$ lies in $H$ (which must be $g_{1}^{2}$, as all other elements of $T$ are primitive). So $g_{1}^{2} \in H$.

Now, take any primitive element $x \in G$, which is part of some generating set $\left\{x, y_{2}, \ldots, y_{n}\right\}$ for $G$ (which must be left-cleaned, otherwise $H$ would contain a primitive element). Using the exact same argument above, with $g_{1}:=x, g_{i}:=y_{i}$ 
for all $2 \leq i \leq n$, we see that if $H$ contains no primitive element then $x^{2} \in H$. So $H$ contains the square of every primitive element.

Lemma 4.3. Let $G$ be any group with $d(G)=n$, and $H$ a subgroup of finite index in $G$ with $[G: H]<3 \cdot 2^{n-1}$. If $H$ contains no primitive elements of $G$, then $H$ is normal in $G$ and $G / H \cong C_{2}^{m}$ for some $m \leq n$.

Proof. Suppose $H$ contains no primitive element. Then, by Lemma 4.2, $H$ contains the square of every primitive element. Set $T:=\left\{g^{2} \mid g\right.$ is primitive in $\left.G\right\}$. Then $T$ is a normal subset of $G$, since the conjugate of a primitive element is again a primitive element (conjugation is an automorphism). Thus $\langle T\rangle \triangleleft G$; the (normal) subgroup generated by all the squares of primitive elements of $G$. So by hypothesis, $T<H$. Now take any generating set $\left\{t_{1}, \ldots, t_{n}\right\}$ for $G$, then $t_{i}^{-1} t_{j}$ is a primitive element, for any pair $i, j$ with $i \neq j$. Thus $t_{i}^{2}, t_{j}^{2},\left(t_{i}^{-1} t_{j}\right)^{2}$ lie in $\langle T\rangle$, and hence so will $t_{i}^{2}\left(t_{i}^{-1} t_{j}\right)^{2} t_{j}^{-2}=\left[t_{i}, t_{j}\right]$. So $G /\langle T\rangle$ is abelian as $\langle T\rangle$ is normal and contains the commutator of every pair in the generating set $\left\{t_{1}, \ldots, t_{n}\right\}$ for $G$. So $\langle T\rangle$, and hence $H$, contain the commutator subgroup $[G, G]$. Thus $H$ is normal in $G$ and $G / H$ is generated by the images of $\left\{t_{1}, \ldots, t_{n}\right\}$, all of which have order 2 in this quotient. So $G / H \cong C_{2}^{m}$ for some $m \leq n$.

We now give the following complete characterisation of finite index subgroups of a group of rank $n$ which contain primitive elements, up to index $3 \cdot 2^{n-1}-1$.

Theorem 4.4. Let $G$ be any group with $d(G)=n$ and let $H$ be a subgroup of finite index in $G$ with $[G: H]<3 \cdot 2^{n-1}$. Then $H$ contains no primitive elements of $G$ if and only if $H$ is normal in $G$ and the quotient $G / H$ is isomorphic to $C_{2}^{n}$, whereupon every coset distinct from $H$ contains a primitive element of $G$.

Proof. First, if $H$ is normal and contains an element $g$ of a generating $n$-tuple for $G$, then the image of this $n$-tuple gives rise to a generating $(n-1)$-tuple of $G / H$, just as in Lemma 4.1 , but $d\left(C_{2}^{n}\right)=n$.

Now suppose that $H$ does not contain a primitive element of $G$ and let $q: G \rightarrow G / H$ be the quotient homomorphism, where we know $H$ is normal in $G$ and $G / H \cong C_{2}^{m}$ for some $m \leq n$ by Lemma 4.3. Given a generating $n$-tuple $\left(g_{1}, \ldots, g_{n}\right)$ for $G$, let $F_{n}$ be the free group on $x_{1}, \ldots, x_{n}$ and set $\theta: F_{n} \rightarrow G$ to be the homomorphism extending the map $x_{i} \mapsto g_{i}$. Note that if we have $k \leq n$ and integers $1 \leq i_{1}<i_{2}<\cdots<i_{k} \leq n$ then $x_{i_{1}} x_{i_{2}} \cdots x_{i_{k}}$ is primitive in $F_{n}$ and $\theta\left(x_{i_{1}} x_{i_{2}} \cdots x_{i_{k}}\right)=g_{i_{1}} g_{i_{2}} \cdots g_{i_{k}}$ is primitive in $G$.

Assume that $m<n$ and consider the map $q \circ \theta: F_{n} \rightarrow C_{2}^{m}$, which factors through $C_{2}^{n}$ via the abelianisation map ab: $F_{n} \rightarrow C_{2}^{n}$ and the map $\psi: C_{2}^{n} \rightarrow C_{2}^{m}$. That is, the following diagram commutes, and all maps are surjections:

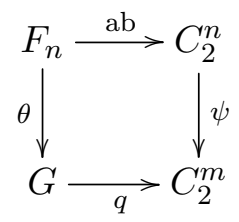

As $\psi$ is now a linear map from an $n$ dimensional vector space over $\mathbb{F}_{2}$ to an $m$ dimensional space, we have a non-trivial element $\left(v_{1}, \ldots, v_{n}\right)$ of $C_{2}^{n}$ in the 
kernel of $\psi$. Now we can assume that each $v_{i}$ takes the value 0 or 1 , so we form the primitive element $x=x_{1}^{v_{1}} x_{2}^{v_{2}} \cdots x_{n}^{v_{n}}$ of $F_{n}$ which maps to the identity under $\psi \circ \mathrm{ab}$, thus $g_{1}^{v_{1}} g_{2}^{v_{2}} \cdots g_{n}^{v_{n}}=\theta(x)$ is a primitive element of $G$ which maps to the identity under $q$ and so is in $H$; a contradiction. Similarly, if $n=m$, then for any $\left(w_{1}, \ldots, w_{n}\right) \in \mathbb{F}_{2}^{n} \backslash\{0\}$ the coset of $H$ in $G$ corresponding to this point contains the primitive element $g_{1}^{w_{1}} g_{2}^{w_{2}} \cdots g_{n}^{w_{n}}$ of $G$.

Thus if $G$ is a group with $d(G)=n$ we have two possibilities: either $C_{2}^{n}$ is not a quotient of $G$ in which case all subgroups of $G$ having index less than $3 \cdot 2^{n-1}$ contain primitive elements, or $G$ surjects to $C_{2}^{n}$ in which case there is a single subgroup of index less than $3 \cdot 2^{n-1}$ which fails to contain a primitive element. The uniqueness in the second case comes about because a homomorphism from a rank $n$ group $G$ to an abelian group of exponent 2 must factor through $G /[G, G] G^{2}$. As $G /\left([G, G] G^{2}\right) \cong C_{2}^{m}$ for $m \leq n$, we see that when $n=m$ any exceptional subgroup must be equal to $[G, G] G^{2}$.

Note that the inequality $[G: H]<3 \cdot 2^{n-1}$ in Theorem 4.4 is somewhat necessary: here is an example of what occurs when the inequality doesn't hold.

Example 4.5. Take the (free) subgroup $H:=\left[F_{n}, F_{n}\right] F_{n}^{2}<F_{n}$ of index $2^{n}$ with no primitive elements of $F_{n}$. Then $H$ itself has several normal subgroups of index 2 , none of which contain primitive elements of $F_{n}$. In $F_{n}$, these subgroups have index $2 \cdot 2^{n}>3 \cdot 2^{n-1}$, so this is not a counterexample to Theorem 4.4.

We remark that the number of subgroups of $F_{n}$ with index less than $2^{n}$ is vast; bounded below by $\left(\left(2^{n}\right) !\right)^{n-1}$ (see [8, Corollary 2.1.2]).

It would be interesting to find a closed form expression for $M(n)$, which we define to be the smallest number $i$ such that $F_{n}$ has a subgroup other than $\left[F_{n}, F_{n}\right] F_{n}^{2}$ of index $i$ which contains no primitive elements. By Theorem 4.4, and the example immediately proceeding it, we have $3 \cdot 2^{n-1} \leq M(n) \leq 2 \cdot 2^{n}$. In particular, consider any quotient map $f: F_{2} \rightarrow S_{3}$; the kernel $N$ of this map has index $6=3 \cdot 2^{2-1}$, and moreover $N$ contains no primitive elements of $F_{2}$ by Lemma 4.1. So $M(2)=6$. Moreover, it is straightforward to see that $M(1)=3$. We do not know $M(n)$ for any other values of $n$.

Our analysis of finite index subgroups of $F_{n}$ containing no primitive elements was motivated by the following result of Parzanchevski and Puder in [11]:

Theorem 4.6 ([11, Corollary 1.5]). The set $P$ of primitive elements in $F_{n}$ is closed in the profinite topology.

Corollary 4.7. Given $F_{n}$, and $w \in F_{n}$ a non-primitive element, there is a finite index subgroup $H<F_{n}$ such that the coset $w H$ does not contain any primitive elements. Taking $w=e$ gives a finite index subgroup with no primitive elements.

We finish by remarking that a recent result of Clifford and Goldstein [6] proves there is an algorithm to determine which finitely generated subgroups of $F_{n}$ contain primitive elements, although they say that they do not expect it to be implemented in practice. One of our overall aims is to give a characterisation of such subgroups that leads to computationally-efficient recognition. A classical result of Whitehead [14] gives an algorithm for determining if an element of $F_{n}$ is primitive; Roig et. al. [12] refine this to a polynomial time algorithm. 


\section{REFERENCES}

[1] A. Blass, Injectivity, Projectivity, and the Axiom of Choice, Trans. Amer. Math. Soc. 255, 31-59, (1979).

[2] J. Button, M. Chiodo, M. Zeron-Medina Laris, Coset intersection graphs, and transversals as generating sets for finitely generated groups, J. González-Meneses et al. (eds.), Extended Abstracts Fall 2012, Trends in Mathematics 5, 29-34, Birkhäuser, (2014).

[3] J. Button, M. Chiodo, M. Zeron-Medina Laris, Coset intersection graphs for groups, Amer. Math. Monthly 121, No. 10, 922-926, (2014).

[4] P. Cameron, Generating a group by a transversal http://www.maths.qmul.ac.uk/ pjc/papers.html

[5] P. Cameron, http://www.maths.qmul.ac.uk/ pjc/oldprob.html

[6] A. Clifford, R. Goldstein, Subgroups of free groups and primitive elements, J. Group Theory 13, 601-611, (2010).

[7] M. J. Evans, Primitive elements in free groups, Proc. Amer. Math. Soc. 106, 313-316, (1989).

[8] A. Lubotzky and D. Segal, Subgroup Growth, Progress in Mathematics 212, Birkhaüser Verlag, Basel, (2003).

[9] G. A. Noskov, Primitive elements in a free group, Matematicheskie Zametki 30, 497-500, (1981).

[10] I. Pak, What do we know about the product replacement algorithm?, Groups and computation, III, 301-347, Ohio State Univ. Math. Res. Inst. Publ. 8, de Gruyter, Berlin, (2001).

[11] O. Parzanchevski, D. Puder, Measure preserving words are primitive, J. Amer. Math. Soc. 28, 63-97, (2015).

[12] A. Roig, E. Ventura, P. Weil, On the complexity of the Whitehead minimization problem, Internat. J. Algebra Comput. 17, No. 8, 1611-1634, (2007).

[13] J. Whiston, Maximal independent generating sets of the symmetric group, J. Algebra 232, 255-268, (2000).

[14] J. H. C. Whitehead, On equivalent sets of elements in a free group, Ann. of Math. 37, No. 4, 782-800, (1936).

Selwyn College, Cambridge

Grange Road, Cambridge, CB3 9DQ, UK

J.O.BuTTON@DPMMS.CAM.AC.UK

Mathematics Department, University of Neuchâtel

Rue Emile-Argand 11, Neuchâtel, CH-2000, SWITZERLAND

MAURICE.CHIODO@UNINE.CH

31 Mariner's Way, Cambridge, CB4 1BN, UK

MARIANOZERON@GMAIL.COM 